\title{
GENÉTICA DE LA RESISTENCIA A ROYA AMARILLA EN PLANTAS ADULTAS DE TRIGO HARINERO
}

\section{GENETICS OF THE YELLOW RUST RESISTANCE IN ADULT PLANTAS OF WHEAT CULTIVARS}

\author{
Óscar M. Villaseñor-Espín ${ }^{1}$, Julio Huerta-Espino ${ }^{2 *}$, S. Gerardo Leyva Mir ${ }^{3}$, Héctor E. Villaseñor-Mir ${ }^{4}$, \\ Ravi P. Singh ${ }^{3}$, J. Sergio Sandoval-Islas ${ }^{1}$ y Eduardo Espitia-Rangel ${ }^{2}$
}

\begin{abstract}
${ }^{1}$ Instituto de Fitosanidad. Colegio de Postgraduados, km 36.5 Carr. México-Texcoco. 56230, Montecillo, Texcoco, Edo. de México. ${ }^{2}$ Campo Experimental Valle de México, Instituto Nacional de Investigaciones Forestales, Agrícolas y Pecuarias. Apdo. Postal 10, Chapingo, Edo. de México. ${ }^{3}$ Centro Internacional de Mejoramiento de Maíz y Trigo. Apdo. Postal 6641. México, D. F. ${ }^{4}$ Departamento de Parasitología, Universidad Autónoma Chapingo.
\end{abstract}

\section{RESUMEN}

La roya lineal amarilla del trigo (Triticum aestivum L.) causada por Puccinia striiformis f. sp. tritici es una enfermedad de creciente importancia en los Valles Altos de México, pues llega a causar pérdidas en el rendimiento mayores a $60 \%$ y deteriora la calidad del grano. Las variedades de trigo harinero 'Juchi F2000', 'Náhuatl F2000' y 'Tlaxcala F2000' fueron liberadas por el Instituto Nacional de Investigaciones Forestales, Agrícolas y Pecuarias en el año 2000 para siembras de temporal o secano, que mostraron diferentes grados de infección por esta enfermedad. Para determinar la genética de la resistencia a roya amarilla de las tres variedades, éstas se cruzaron con la variedad susceptible 'Avocet$Y_{r A}{ }^{\prime}$. Los progenitores y 148 familias $F_{3}$ por cruza se evaluaron durante el Verano 2002 en Toluca, Estado de México, bajo una incidencia natural de roya amarilla. Con los resultados de las familias $F_{3}$ se determinó que la resistencia de planta adulta a roya amarilla en 'Juchi F2000' está regalada por tres genes menores de efectos aditivos, y que en 'Náhuatl F2000' y 'Tlaxcala F2000' está condicionada por tres o cuatro genes de la misma naturaleza. Un gen en común en las tres variedades fue $\operatorname{Yr18}$, que confiere resistencia parcial de planta adulta a roya amarilla. La progenie de 'Náhuatl F2000' manifestó mayor nivel de resistencia, lo que indica fue contiene más genes o genes diferentes y más efectivos; por ello, a través de una cruza triple entre las tres variedades podría aumentarse el nivel de resistencia a roya amarilla.

Palabras clave: Triticum aestivum L. Puccinia striiformis f. sp. Tritici, herencia de resistencia, genes menores.

\section{SUMMARY}

Wheat (Triticum aestivum L.) yellow rusts caused by Puccinia striiformis f. sp. tritici is a disease that in the High Valleys of México is growing in importance. This disease may cause up to $60 \%$ of yield losses and diminish the industrial quality of the grain. 'Juchi F2000', 'Náhuatl F2000' and 'Tlaxcala F2000', are three varieties of bread wheat released in 2000 by the National Institute of Forestry, Husbandry and Agricultural Research for planting in rainfed areas. During their evaluation these varieties showed different responses tho the disease In order to determine the genetics of the resistance to yellow rust in these three varieties, they were crossed to the susceptible variety 'Avocet- $\mathrm{YrA}^{\prime}$ '. The parents and $148 \mathrm{~F}_{3}$ families per cross were evaluated at Toluca, México during the Summer 2002 under a natural incidence of yellow rust. The results of the $F_{3}$ families allowed to infer that the adult plant resistance in 'Juchi F2000' was conditioned by three minor genes of additive effects. In 'Náhuatl F2000' and 'Tlaxcala F2000' three to four genes of the same nature were identitied. A common gene in the three varieties was $\mathrm{Yr18}$, which confers partial adult plant resistance to yellow rust. 'Náhuatl F2000' progeny showed higher levels of resistance, thus indicating that it has a higher number of resistance genes. The intercross of these three varieties may allow to increase the level of yellow rust resistance.

Index words: Triticum aestivum .Puccinia striiformis f. sp. tritici, inheritance resistance, minor genes.

\section{INTRODUCCIÓN}

En los Valles Altos de México, que comprenden regiones templadas de los Estados de México, Tlaxcala, Hidalgo, Puebla, Durango y Morelos, se siembran más de 150 mil hectáreas de trigo (Triticum aestrivum L.) de temporal o secano durante el verano (SIAP, 2008). En estas áreas temporaleras se presentan condiciones favorables de humedad (mayor de $70 \%$ ) y temperatura $\left(15-20{ }^{\circ} \mathrm{C}\right.$ ) para la incidencia de roya amarilla (Puccinia striiformis f. sp. tritici), enfermedad que cuando ataca de manera severa en variedades susceptibles llega a causar pérdidas del rendimiento mayores de $60 \%$ ) y deteriora fuertemente la calidad del grano (Huerta y Singh, 2000).

El mejoramiento genético para lograr resistencia a esta enfermedad ha sido el método más eficiente en su control (Singh et al., 2002). Biffen (1905), uno de los primeros investigadores que estudió la genética de la 
herencia de infección al ataque de roya amarilla, reportó que dicha resistencia es monogénica y de carácter recesivo. Con el redescubrimiento de las leyes genéticas de la herencia y el descubrimiento de que la resistencia a royas es un carácter de herencia simple, se han realizado numerosos trabajos sobre la herencia de la resistencia a roya amarilla, principalmente para identificar genes mayores. La mayoría de variedades resistentes a roya amarilla han tenido como fuente de resistencia a genes de herencia simple, pero la pérdida continua del efecto de estos genes o de sus combinaciones, ha obligado a buscar alternativas en el manejo de la resistencia no específica a este hongo, debido a que este patógeno tiene la capacidad de mutar y evolucionar hacia mayor virulencia, lo que ocasiona que se rompa rápidamente la resistencia.

Una alternativa para lograr mayor durabilidad de la resistencia a roya amarilla es mediante la formación de genotipos de trigo que posean resistencia durable, basada en genes que confieren resistencia de desarrollo lento de la enfermedad (slow rusting); el efecto de un gen de resistencia de desarrollo lento en el progreso de la enfermedad fluctúa de pequeño a moderado, pero la combinación de dos o más genes de efectos aditivos resulta en un alto nivel de resistencia (Singh y Rajaram, 1994). Las variedades de trigo presentan genes de planta adulta, cuyos efectos son menores pero de acción aditiva (Singh y Dubin, 1997; Singh et al., 2002). Según Milus y Line (1986a y 1986b), las variedades de trigo invernal 'Gaines', 'Nugaines' y 'Luke' exhiben resistencia durable a Puccinia striiformis f. sp. tritici más efectiva en planta adulta y a temperaturas mayores a $25{ }^{\circ} \mathrm{C}$ que a temperaturas menores a $15{ }^{\circ} \mathrm{C}$, que es lo mas común; estos autores concluyeron que en cada variedad la resistencia fue condicionada al menos por dos ó tres genes menores de efectos aditivos.

Los estudios de herencia realizados en los últimos años en variedades mexicanas de trigo indican que los niveles moderados de resistencia en 'Pénjamo T62', 'Lerma Rojo S64', 'Nacozari M76' y 'Tesia F79' están condicionados por la acción monogénica del gen de planta adulta $\operatorname{Yr} 18$ que confiere resistencia parcial (Singh y Rajaram, 1994). Singh y Dubin (1997) y Singh et al. (2000) encontraron que las combinaciones de $\operatorname{Yr} 18$ más dos o tres genes de la misma naturaleza inducen niveles aceptables de resistencia, y que a mayor número de genes de efecto aditivo la resistencia es más estable en cualquier ambiente que presente roya amarilla. En variedades como 'Pavón F76' y 'Huites F95' que no poseen el gen Yr18, también es posible reducir los niveles de infección mediante otros genes que igualmente inducen este tipo de resistencia, como Yr29 y Yr3 (Singh et al., 2001, 2003).
Se ha reportado que no existen efectos negativos en el rendimiento al acumular mayor número de genes menores de acción aditiva, de forma que se pueden conjuntar resistencia a roya amarilla y a roya de la hoja con alto potencial de rendimiento (Huerta y Singh, 2000). En México la primera variedad de trigo liberada para siembras de temporal con este tipo de resistencia fue 'Zacatecaz VT74', seguida de 'Romoga F96' cuya resistencia permanece efectiva aún cuando se manifiesten varias razas de roya amarilla. Otras variedades para siembras de temporal como 'Zacatecas VT74', 'Pavón F76' y 'México M82' han mantenido niveles aceptables de resistencia, pero no así las variedades 'Temporalera', 'Gálvez M86' y 'Batán F96' que actualmente son susceptibles. Las variedades de trigo para siembras de temporal 'Juchi F2000', 'Náhuatl F2000', y 'Tlaxcala F2000' mantienen resistencia a las diferentes razas de roya amarilla que se encuentran en el país, pero se desconoce el número de genes que determina esta resistencia. En estas tres variedades se ha postulado la presencia del gen $\mathrm{Yr} 18$ y otros genes menores de efectos aditivos, pero no se tiene la certeza si $\operatorname{Yr} 18$ se encuentra presente y cuántos mas lo acompañan (Villaseñor et al., 2003).

Para lograr resistencia durable a roya amarilla en las futuras variedades es necesario conjuntar de cuatro a cinco genes de efectos menores de acción génica aditiva, lo que se conoce como resistencia cercana a la inmunidad. La manera de conjuntar este número de genes es a través del mejoramiento genético (Singh et al., 2000). El objetivo de esta investigación fue determinar la herencia de la resistencia de planta adulta a roya amarilla de las tres variedades de trigo para temporal, 'Juchi F2000', 'Náhuatl F2000' y 'Tlaxcala F2000'.

\section{MATERIALES Y MÉTODOS}

El trabajo se efectuó durante los veranos del 2000 al 2002. Se hicieron tres cruzas entre el progenitor susceptible 'Avocet-YrA' (AOC-YrA) con cada una de las variedades resistentes 'Juchi F2000', 'Náhuatl F2000' y 'Tlaxcala F2000', empleadas como progenitores masculinos. En el Cuadro 1 se muestran los genotipos y sus progenitores. Las cruzas se hicieron durante el Verano 2000 en el Batán Texcoco, Estado de México. La generación $\mathrm{F}_{1}$ de cada cruza se sembró en campo de forma mateada durante el ciclo Otoño-Invierno 2000-2001 en Ciudad Obregón, Sonora. De cada cruza se cosecharon cinco plantas al azar, tres de las cuales dieron origen a la generación $\mathrm{F}_{2}$ y las dos restantes se guardaron como reserva. Las progenies de cada planta $F_{1}$ fueron sembradas en forma mateada en el Verano 2001 para cosechar en forma aleatoria 50 plantas $\mathrm{F}_{2}$ de cada una de las tres plantas que conformaron a cada cruza. Para la obtención 
de las familias $F_{3}$, las poblaciones $F_{2}$ de cada cruza se inspeccionaron visualmente, y cuando no había discrepancia en las progenies de las tres distintas plantas $F_{1}$ se cosecharon al azar 50 plantas individuales de cada una de las tres plantas por cruza, para la obtención de 148 familias $F_{3}$.

Cuadro 1. Cruza e historia de selección de las cuatro variedades de trigo utilizadas en el estudio durante el verano de 2002 en Toluca, Estado de México.

\begin{tabular}{ll}
\hline Variedad & Cruza y genealogía \\
\hline 'Juchi F2000' & KITE/BOW//ROMOGA \\
& TC920338-S-9C-04R-1C-0R-1C-0R \\
'Náhuatl F2000' & E7408/PAM//HORK/PF73226/3/ \\
& URES/4/OPATA/5/OPATA/BOW \\
& CMBW89Y00804-0TOPM-9R-0C-2R- \\
& 3C-0R \\
& ZACATECAS/ROMOGA \\
'Tlaxcala F2000' & TC920248-S-34C-06R-1C-0R-1C-0R \\
& WW119/WW151//EGRET \\
'Avocet-YrA' & -YrA-30Y \\
\hline
\end{tabular}

Fuente: Villaseñor et al. (2003) y Huerta-Espino et al. (2002).

Durante el desarrollo de las poblaciones todas las cruzas y generaciones se protegieron con la aplicación del fungicida Tebuconazol (Folicur®) para controlar la enfermedad y evitar la pérdida de plantas susceptibles. Las familias $F_{3}$ de cada cruza se evaluaron en el Verano 2002 en un campo experimental en Toluca, Estado de México. Los progenitores y cada una de las 148 familias $\mathrm{F}_{3}$ de cada cruza se sembraron en surcos dobles de $1 \mathrm{~m}$ de largo separados a $80 \mathrm{~cm}$; se emplearon dos repeticiones para minimizar escapes, pero en el análisis de datos sólo se uso una repetición porque no hubo diferencias significativas entre repeticiones.

El análisis genético se basó en la frecuencia de familias resistentes, susceptibles y segregantes en un tipo de herencia cualitativa o mendeliana. Para lograr alta incidencia de la enfermedad, el experimento se rodeó con un bordo de la variedad susceptible 'Morocco', la cual actuó como fuente de inoculo; al final de cada parcela, en franjas alternas también se sembraron surcos de la misma variedad. La evaluación y clasificación de las familias $\mathrm{F}_{3}$ por su respuesta a la roya amarilla se efectuó bajo incidencia natural del hongo. Para registrar la respuesta a la roya amarilla en las familias se hicieron dos evaluaciones de la enfermedad, la primera cuando el progenitor $\mathrm{F}_{3}$ susceptible alcanzó de 90 a $100 \%$ de severidad en la hoja bandera, y la segunda $7 \mathrm{~d}$. después.
En todas las familias de cada cruza se estimó el porcentaje de severidad en la hoja bandera, de acuerdo con la escala modificada de Cobb (Peterson et al., 1948); para las familias heterocigóticas, identificadas por su segregación, se estimó el promedio de infección de cinco hojas, mediante la misma escala. También se evaluó la $F_{1}$ de cada cruza de la misma forma que las familias $F_{3}$. Sin embargo, en la $\mathrm{F}_{1}$ se tomaron tres lecturas para observar el desarrollo de la enfermedad (Cuadro 2). Una vez hecha la evaluación, las familias $\mathrm{F}_{3}$ de cada cruza se clasificaron en cuatro grupos. En el Grupo 1 se incluyeron familias homocigóticas con respuesta similar al progenitor resistente; en el Grupo 2 se ubicaron a las familias homocigóticas con respuesta similar al progenitor susceptible; en el Grupo 3 se incluyeron las familias heterocigóticas que segregaron plantas resistentes como el progenitor resistente, hasta un porcentaje de severidad de nivel intermedio pero sin plantas similares al progenitor susceptible; y en el Grupo 4 se clasificaron las familias heterocigóticas que segregaron plantas con resistencia semejante al progenitor resistente, plantas con resistencia del tipo intermedio y plantas tan susceptibles como el progenitor susceptible. Con las frecuencias observadas y esperadas en ambas evaluaciones, se realizaron pruebas de $\mathcal{X}^{2}$.

\section{RESULTADOS Y DISCUSIÓN}

Las condiciones de temperatura $\left(\begin{array}{lllll}10 & \text { a } & 25 & { }^{\circ} \mathrm{C}\end{array}\right)$ y humedad (mayor a $70 \%$ ) fueron favorables para el desarrollo de la enfermedad, la cual alcanzó altos niveles de infección en la hoja bandera (100 \% de infección) en el progenitor susceptible, lo que permitió la evaluación y clasificación de la $F_{1}$, de las familias $F_{3}$ y de los progenitores. La $\mathrm{F}_{1}$ de las cruzas entre variedades resistentes y el progenitor susceptible indicó que la resistencia a roya amarilla fue parcialmente dominante, como se muestra en el Cuadro 2. Al analizar el comportamiento de las familias $\mathrm{F}_{3}$ respecto a los niveles de infección, y al clasificarlas en resistentes, segregantes y susceptibles, no se observó una distribución discreta de clases que pudiese indicar la presencia de genes de resistencia de efectos mayores o factores de resistencia de dominancia completa; lo que se observó fue una variación continua de las familias $F_{3}$. En la Figura 1 se muestra la distribución de familias $\mathrm{F}_{3}$ en cada cruza. 

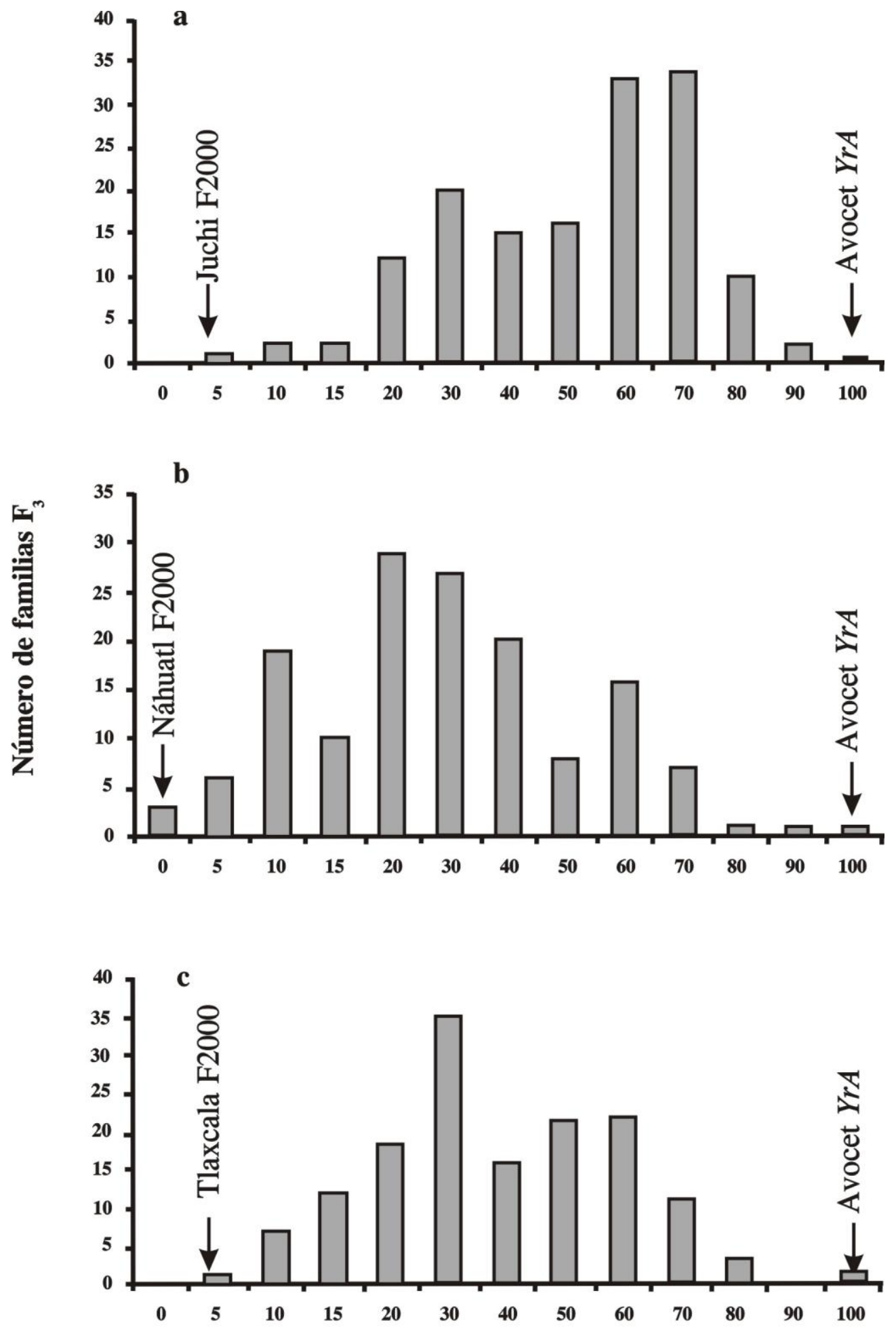

Severidad de roya amarilla $(\%)$

Figura 1. Distribución de familias F3 de las cruzas entre el progenitor susceptible 'Avocet-Yr'A con las variedades resistentes: a) 'Juchi F2000', b) 'Náhuatl F2000', y c) 'Tlaxcala F2000'. 
Cuadro 2. Porcentaje de infección y reacción (1) a roya amarilla de los progenitores y $\mathbf{F}_{1}$ de las respectivas cruzas resistente $\mathcal{X}$ susceptible en tres fechas de evaluación.

\begin{tabular}{lccc}
\hline Progenitor o Cruza & 2 de agosto & 7 de agosto & 22 de agosto \\
\hline 'Avocet- $Y r A$ ' & $90 \mathrm{~S}$ & $100 \mathrm{~S}$ & $100 \mathrm{~S}$ \\
'Juchi F2000' & $10 \mathrm{MR}$ & $15 \mathrm{MR}$ & $20 \mathrm{MR}$ \\
'AOC-YrA/ Juchi F2000' & $70 \mathrm{~S}$ & $80 \mathrm{MS}$ & $90 \mathrm{MS}$ \\
'Náhuatl F2000' & $1 \mathrm{MR}$ & $5 \mathrm{R}$ & $10 \mathrm{R}$ \\
'AOC-YrA/ Náhuatl F2000' & $30 \mathrm{M}$ & $50 \mathrm{M}$ & $60 \mathrm{M}$ \\
'Tlaxcala F2000' & $1 \mathrm{MR}$ & $5 \mathrm{MR}$ & $10 \mathrm{MR}$ \\
'AOC-YrA/ Tlaxcala F2000' & $50 \mathrm{MS}$ & $60 \mathrm{MS}$ & $70 \mathrm{MS}$ \\
\hline
\end{tabular}

(1) Reacción de la planta a la enfermedad: S = Susceptible; MR = Moderadamente resistente; MS = Moderadamente susceptible; R= Resistente.

El criterio para determinar el número de genes se basó en el número de familias homocigóticas susceptibles, las cuales son más fáciles de identificar en campo. Bajo el supuesto de que la virulencia del patógeno es recesiva y que la resistencia en la planta es dominante (Roelfs y Groth, 1988), el número de genes implicados en la resistencia se determinó con base en las frecuencias observadas de las familias clasificadas en el Grupo 2. Entonces, si la resistencia estuviera controlada por dos genes la frecuencias esperadas de familias homocigóticas susceptibles sería de $(6.25 \%)$ 1/16; si fuera condicionada por tres genes, la proporción sería de 1/64 (1.6\%), y si estuviera controlada por cuatro genes, la proporción sería de $1 / 256$, equivalente a $0.4 \%$ (Cuadro 3 ).

Cuadro 3. Frecuencias esperadas (\%) en los cuatro grupos de clasificación de familias $F_{3}$ en cruzas susceptibles por resistentes, cuando la resistencia está condicionada por genes menores de efectos aditivos.

\begin{tabular}{ccccc}
\hline Núm. de genes & Grupo 1 & Grupo 2 & Grupo 3 & Grupo 4 \\
\hline 2 & 6.3 & 6.3 & 37.5 & 50.0 \\
3 & 1.6 & 1.6 & 56.3 & 40.6 \\
4 & 0.4 & 0.4 & 68.0 & 31.3 \\
5 & 0.1 & 0.1 & 76.2 & 23.6
\end{tabular}

Grupo 1 = Familias homocigóticas resistentes; Grupo 2 = Familias homocigóticas susceptibles; Grupo $3=$ Familias heterocigóticas con plantas resistentes y plantas intermedias; Grupo $4=$ Familias heterocigóticas con plantas resistentes, plantas intermedias y susceptibles.

Con base en las frecuencias esperadas y observadas (Cuadro 4), mediante la prueba de $\mathcal{X}^{2}$ se determinó que tres es el número mínimo de genes de efecto aditivo que controlan la resistencia en las tres variedades. Como en la van "Náhuatl F2000" se incrementó la frecuencia de familias de tipo intermedio o Grupo 3 con respecto a las familias del Grupo 4, probablemente en esta variedad el número de genes sea mayor (Figura 1). Lo anterior se evidencia también en el mayor nivel de resistencia en la F1. Lo anterior se ha observado en genotipos cuyo número de genes de efecto aditivo es mayor, con niveles de resistencia en la F1 que en algunos casos es similar al progenitor resistente (Singh et al., 2000).

Resultados similares reportaron Navabi et al. (2004) y Singh et al (2000 y 2002 en trigo; además, se confirma que para alcanzar mayores niveles de resistencia es necesario acumular mayor número de genes de efecto aditivo, como en 'Náhuatl F2000'. En las tres variedades se ha postulado la presencia (Huerta-Espino et al., 2002) del gen $\mathrm{Yr} 18 / \mathrm{Lr} 34$ ligado o pleiotrópico a la necrosis de la punta de la hoja (leaf tip necrosis $=l t n$ ), gen que reduce los niveles de infección de la enfermedad pero cuya acción individual es insuficiente porque los niveles de desarrollo de la enfermedad son mayores a $70 \%$. En el presente estudio fue evidente la necrosis de la punta de la hoja, por lo que se confirmó que $\operatorname{Yr} 18$ es uno de los genes presentes en las variedades 'Juchi F2000', 'Náhuatl F2000' y 'Tlaxcala F2000'. Este gen se considera de naturaleza durable y con efecto sinergístico al interactuar con otros genes de efectos aditivos, pues puede mejorar el nivel de resistencia en forma individual; estos genes en un tiempo se denominaron complejo Yr18 (Singh y Rajaram,1992, 1994; Singh y Dubin, 1997).

Al analizar los progenitores (Cuadro 1) se puede deducir que en 'Juchi F2000' el gen Yr18 proviene de 'Romoga F96', variedad que fue evaluada durante 10 años (1986 - 1996) y desde entonces mostró nivel aceptable de resistencia a roya amarilla, aún cuando la frecuencia de poblaciones del patógeno ha cambiado a formas más virulentas. Este progenitor, al igual que 'Zacatecas VT74' posee el gene $\operatorname{Yr} 18$ por lo que en la cruza 'Zacatecas VT74/Romoga F96' este gen se encontró en estado homocigótico al igual que en la variedad 'Tlaxcala F2000'. En 'Náhuatl F2000' lo más probable es que $\operatorname{Yr} 18$ provenga de la var. 'Opata', aunque otros progenitores implicados en la cruza, como 'PAM' y 'HORK', también poseen el gen (Villaseñor et al., 2003). 
Cuadro 4. Distribución y frecuencia relativa de familias $F_{3}$ en cuatro grupos de las cruzas entre el progenitor susceptible ('Avocet- $\left.Y r^{\prime} A\right)$ y los progenitores resistentes ('Juchi F2000', 'Náhuatl F2000' y 'Tlaxcala F2000') en Toluca, Estado de México durante el Verano 2002.

\begin{tabular}{|c|c|c|c|c|c|c|c|c|c|c|}
\hline \multirow{3}{*}{ Cruza } & \multicolumn{8}{|c|}{ Grupo } & \multirow[b]{3}{*}{ Núm. de genes } & \multirow[b]{3}{*}{$\mathrm{X}^{2}$} \\
\hline & \multicolumn{4}{|c|}{ Núm. de familias $F_{3}$} & \multicolumn{4}{|c|}{ Frecuencia relativa } & & \\
\hline & 1 & 2 & 3 & 4 & 1 & 2 & 3 & 4 & & \\
\hline 'AOC-YrA / Juchi' & 3 & 1 & 90 & 54 & 2.0 & 0.6 & 60.8 & 36.4 & 3 & 1.5 \\
\hline 'AOC-YrA / Tlaxcala' & 1 & 2 & 89 & 56 & 0.6 & 1.3 & 60.1 & 37.8 & 3 & 1.1 \\
\hline
\end{tabular}

$3 \mathrm{gl}, \alpha=0.05, \chi^{2} \mathrm{t}=7.815$

La presencia de más de tres genes de resistencia a roya amarilla en las variedades liberadas durante el 2000 indica que es posible conjuntar alto potencial de rendimiento y buenos niveles de resistencia, con calidad de grano y otras características agronómicas deseables. Ante una población de roya amarilla que se mantiene en cambio constante, la mejor forma de asegurar la sustentabilidad del trigo de temporal es mediante el uso de variedades con resistencia durable y evitar el uso de variedades con resistencia específica, para lo cual es importante que en el mejoramiento genético se enfatice en el uso de resistencia no específica o resistencia de desarrollo lento de la roya, que ha probado ser de tipo durable. Es necesario entonces diversificar las fuentes de resistencia para recombinar diferentes genes y así alcanzar una resistencia más estable en los diferentes ambientes.

Los resultados de las cruzas en $\mathrm{F}_{1}$ y de las familias $\mathrm{F}_{3}$ indica que al menos la variedad 'Náhuatl F2000' posee mayor número de genes que las otras dos variedades; no obstante, es necesario analizar si las tres variedades poseen genes diferentes, lo cual requiere cruzarlas entre sí y confirmar que las tres poseen el gen Yr18, además de determinar cuántos genes son diferentes. En caso de que haya por lo menos un gen de diferencia entre ellas, entonces se puede incrementar el nivel de resistencia al entrecruzar estas variedades, y mantener como base al gen Yr18. El entrecruzamiento o la combinación de estas tres variedades pueden conjuntar no solamente mayores niveles de resistencia, sino también mayor rendimiento y calidad, puesto que individualmente las tres variedades son de alto potencial de rendimiento y buena calidad (Villaseñor et al., 2003).

\section{CONCLUSIONES}

Con base en los resultados de las familias $\mathrm{F}_{3}$, se concluye que la resistencia de planta adulta a roya amarilla en la variedad 'Juchi F2000' está determinada por tres genes de efectos aditivos. En las variedades 'Náhuatl F2000' y 'Tlaxcala F2000' se identificaron entre tres y cuatro genes. El gen $\mathrm{Yr} 18$ es uno de los genes de resistencia que poseen estas tres variedades de trigo harinero de temporal liberadas durante el 2000. Se considera que al menos 'Náhuatl F2000' posee mayor número de genes o cuando menos un gen diferente que le confiere mayor nivel de resistencia, lo que permitiría alcanzar mayor resistencia durable a roya amarilla en otras variedades que se cruzaran con ella.

\section{AGRADECIMIENTOS}

Al CONACYT (Proyectos 34715-B y 12163) por el financiamiento otorgado para la realización de la presente investigación.

\section{BIBLIOGRAFIA}

Biffen R H (1905) Mendel's laws of inheritance and wheat breeding. J. Agric. Sci. 1:4-48.

Huerta E J, R P Singh (2000) Las royas del trigo. In: El Trigo de Temporal en México. H E Villaseñor y E Espitia R (eds). Libro Técnico No. 1. Chapingo, Edo. de México. SAGAR, INIFAP, CIRCE, CEVAMEX. pp:231-251.

Huerta E J, H E Villaseñor, E Espitia R, S G Leyva M, R P Singh (2002) Análisis de la resistencia a la roya de la hoja en trigos harineros para temporal. Rev. Fitotec. Mex.25:161-169.

Milus E A, R F Line (1986a) Number of genes controlling hightemperature, adult plant resistance to stripe rust in wheat. Phytopathology 76:93-96.

Milus E A, R F Line (1986b) Gene action for inheritance of durable, high-temperature, adult plant resistance to stripe rust in wheat. Phytopathology 76:435-441.

Navabi A, R P Singh, J P Tewari, K G Briggs (2004) Inheritance of high levels of adult plant resistance to stripe rust in five spring wheat genotypes. Crop Sci. 44:1156-1162.

Peterson R F, A B Campbell, A E Hannah (1948) A diagrammatic scale for estimating rust intensity of leaves and stem of cereals. Can. J. Res. Section C, 26:496-500. 
Roelfs A P, J V Groth (1988) Puccinia graminis f. sp. tritici black stem rust of Triticum spp. In: Genetics of Plant Pathogenic Fungi. Advances in Plant Pathology Vol. 6 GS Sidhu (ed). pp: 345-361.

SIAP (2008) Avance de Siembras y Cosechas 2008. Disponible en: www.siap.sagarpa.gob.mx. (Enero 2009).

Singh R P, S Rajaram (1992) Genetics of adult-plant resistance to leaf rust in 'Frontana' and three CIMMYT wheats. Genome 35:2431.

Singh R P, S Rajaram (1994) Genetics of adult plant resistance to stripe rust in ten spring bread wheats. Euphytica 72:1-7.

Singh R P, J H Dubin (1997) Sustainable control of wheat diseases in Mexico. In: Mem. First International Wheat Symposium. Cd. Obregon, Sonora, Mexico. pp:93-103.

Singh R P, J Huerta-Espino, S Rajaram (2000). Achieving nearimmunity to leaf and stripe rust in wheat by combining slow rusting resistance genes. Acta Phytopathol. Entomol. Hungarica 35:133-139.

Singh R.P, J Huerta-Espino, M William (2001) Slow rusting genes based resistance to leaf and yellow rust in wheat: Genetics and
Breeding at CIMMYT. In: Wheat Breeding Society of Australia, Inc. 10th Assembly Proc. R Eastwood, G Hollamby, T Rathjen, N Gororo (eds). 16-21 Sept 2001. Mildura, Australia. pp:103108

Singh R P, J Huerta-Espino, A P Roelfs (2002) The wheat rust. In Bread Wheat. B C Curtis, S Rajaram, M H Gomez (eds). FAO. Roma, Italia. pp:246-271.

Singh R P, J Huerta-Espino, M William (2003) Resistencia durable a roya de la hoja y roya amarilla del trigo: Genética y mejoramiento en el CIMMYT. In: Estrategias y Metodologías Utilizadas en el Mejoramiento de Trigo. M M Kohlí, M Díaz, M Castro (eds). Seminario Internacional, La Estanzuela, Uruguay. CIMMYT-INIA. pp:109-118.

Villaseñor M H E, E R Espitia, J Huerta E, C Ortiz (2003) Variedades de trigo F2000, una opción para las siembras de temporal en el Estado de México. Folleto Técnico No. 15. SAGARPA, INIFAP, CEVAMEX. Chapingo, México. 36 p. 